\title{
TV/Series
}

$18 \mid 2020$

Séries et espace

\section{Downton Abbey, ou comment représenter les rapports sociaux à travers la demeure et son espace}

\section{Victor Faingnaert}

\section{OpenEdition}

\author{
Journals
}

Édition électronique

URL : http://journals.openedition.org/tvseries/4776

DOI : 10.4000/tvseries.4776

ISSN : 2266-0909

Éditeur

GRIC - Groupe de recherche Identités et Cultures

Référence électronique

Victor Faingnaert, «Downton Abbey, ou comment représenter les rapports sociaux à travers la demeure et son espace »,TV/Series [En ligne], 18 | 2020, mis en ligne le 15 septembre 2020, consulté le 10 décembre 2020. URL : http://journals.openedition.org/tvseries/4776 ; DOI : https://doi.org/ $10.4000 /$ tvseries.4776

Ce document a été généré automatiquement le 10 décembre 2020.

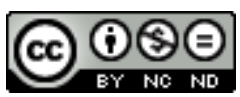

$T V /$ Series est mis à disposition selon les termes de la licence Creative Commons Attribution - Pas d'Utilisation Commerciale - Pas de Modification 4.0 International. 


\title{
Downton Abbey, ou comment représenter les rapports sociaux à travers la demeure et son espace
}

\author{
Victor Faingnaert
}

1 Au fil de ses six saisons, la série Downton Abbey de Julian Fellowes (ITV1, 2010 - 2015) a raconté l'histoire, au début du XXe siècle, de la famille Crawley, issue de l'aristocratie britannique, et de ses domestiques. En plus de rassembler plus de 9 millions de téléspectateurs britanniques par épisode ${ }^{1}$ et à peu près autant lors des diffusions américaines $^{2}$, la série s'est également imposée par son accueil critique, s'inscrivant même dans le Guinness Book des records comme la série britannique la plus acclamée de l'histoire ${ }^{3}$. À tous ces records s'ajoutent une reconnaissance institutionnelle et de nombreuses récompenses telles que 10 nominations et 3 Golden Globes ou 12 Emmy Awards pour 59 nominations ${ }^{4}$.

2 Cet engouement autour de Downton Abbey lui confère une place bien particulière dans le paysage culturel britannique. Par exemple, en 2013, un projet de loi s'est vu surnommé «Downton Abbey Law». De son véritable nom, l'Equality (Titles) Bill aurait permis aux femmes aînées d'hériter des titres héréditaires et des pairies. Ce problème est au cœur des premières saisons de la série et a ainsi permis de soulever cette question au Parlement et dans la société en général. Cette place particulière dans le paysage britannique se matérialise également par une immense entreprise touristique au château de Highclere, qui héberge des concerts, des mariages et de multiples cérémonies en rapport avec la série. Ce château appartient aux Carnarvon, de très proches amis du showrunner ${ }^{5}$, où une partie de la série est tournée, tant pour les décors extérieurs, qu'intérieurs. Une partie du scénario est également librement inspiré de l'histoire des Carnarvon au début du XXe siècle. Enfin on ne compte plus le nombre d'ouvrages sur la série, qu'ils soient officiels ou non ${ }^{6}$, traitant de making-of, de suppléments pour certaines saisons, mais également de philosophie ou de guide de bonnes manières ${ }^{7}$. 
3 Les critiques de Downton Abbey sont plus ou moins unanimes sur la question : la série offre une vision conservatrice de la société britannique ${ }^{8}$. La série s'illustre comme une digne héritière des films heritage $e^{9}$ des années 1980 et 1990 et promeut des valeurs datées ainsi qu'un ordre social immuable et rigide ${ }^{10}$. De nombreuses critiques ont insisté sur l'origine familiale du showrunner Julian Fellowes. En effet ce dernier, très ami avec les Carnarvon, est également Lord à vie, et siège à ce titre à la chambre des Lords en tant que membre du parti conservateur. Il est normal d'analyser son œuvre au prisme de cet engagement politique et de sa vie puisque la série est extrêmement liée à son histoire personnelle. Par exemple, le titre vient du Downton Agricultural College, fondé par son arrière-grand-père, John Wrightson ${ }^{11}$, et Fellowes assume également qu'une grande partie des personnages sont inspirés soit de sa famille, soit de personnes rencontrées ${ }^{12}$. Et on peut donc voir dans la série une représentation de son sentiment de classe, d'appartenance à un milieu, et une défense de ce mode de vie. Tout cela peut nourrir une analyse de la représentation de cet espace dans la série. On peut alors préciser que le genre même de heritage costume drama repose sur la présentation d'un mode de vie perdu, principalement autour de la country house, qu'incarne parfaitement la demeure de Downton.

Nous considérons que les fictions historiques audiovisuelles ne sont pas uniquement des divertissements, des produits culturels nous renseignant sur leur contexte de création, mais également une forme de récit historique. Elles sont alors à analyser de la même façon que l'on peut le faire pour les différents types d'écritures historiques. En s'inspirant, alors, de la tentative fondatrice de Hayden White ${ }^{13}$, on peut questionner les types d'écritures que proposent les séries télévisées historiques. La façon de raconter l'espace, de le filmer et de lui donner un sens est un des points clefs d'une série comme Downton Abbey qui se construit avec les codes du upstairs downstairs, un genre tiré de la série éponyme diffusée entre 1971 et $1975^{14}$, dans lequel, comme le titre le suppose, le lieu et l'espace jouent un rôle primordial ${ }^{15}$. Par cette opposition entre "étages " les œuvres qui s'illustrent dans ce genre mettent en scènes des espaces sociaux. La façon de les mettre en scène, ou ici, de les filmer, donne une vision et un sens à l'histoire du Royaume-Uni du début du XXe siècle. Analyser ainsi ce récit sériel autour de ce lieu et de cet espace permet de croiser les différentes problématiques et sujets de la série et d'en faire ressortir des significations profondes qui la construisent.

Nous analyserons dans un premier temps la construction de la maison, à la fois comme personnage et comme miroir de la société britannique du début $\mathrm{du} \mathrm{XX}^{\mathrm{e}}$ siècle. Nous poursuivrons en tentant d'analyser le sens d'une série conservatrice, à travers le rapport à la maison, à la demeure familiale, et aux différents espaces qui la composent. Nous verrons de ce fait comment ce sentiment nostalgique ${ }^{16}$ se retrouve dans la série au-delà du scénario, mais également dans l'intention même du réalisateur et comment cela se traduit dans le choix des espaces et la façon de les mettre en scène. Nous poursuivrons, en dépassant ce premier prisme d'analyse, pour montrer la façon dont la série, par sa demeure et ce qu'elle représente, regorge de tentatives et de possibilités d'émancipation ${ }^{17}$ pour les personnages. Nous verrons ainsi la façon dont la demeure, filmée comme un lieu de maintien d'un ordre social, laisse en réalité suffisamment de place à certains personnages pour lutter contre cet ordre social et parvenir à s'en éloigner, même temporairement. 


\section{Downton, personnage et miroir}

\section{La personnification du manoir}

6 La demeure est l'objet de la série, celle dont on doit s'occuper, et à ce titre elle est liée aux Crawley comme n'importe quel membre de la famille, elle est donc filmée et mise en scène à la manière de personnages humains. Le titre de la série fait ainsi porter l'attention non pas tant sur la famille Crawley directement, mais sur le lieu où ils demeurent et où l'essentiel de l'action se déroule. La maison, la demeure familiale, devient ainsi l'héroïne de la série, dont les personnages ne sont alors que des résidents temporaires, de la même manière qu'une véritable lignée nobiliaire est tributaire d'un lieu. Cette question apparaît ainsi dès le générique : ce sont les espaces de la demeure et les terres alentour qui sont mises en scène, avec les noms du casting s'inscrivant au fur et à mesure. Plusieurs silhouettes interviennent dans ce générique, ce sont des personnages anonymes, une silhouette représentant une femme de chambre (Figures 1 et 2), une main représentant un majordome ou un valet de pied (Figures 3 et 4). Tandis que les lieux sont représentés par l'intermédiaire d'un objet qui est emblématique de cet espace, le courrier et l'escalier pour la salle des serviteurs, la table à dîner pour la salle à manger, le lustre pour le salon. Les lieux et les espaces sont caractérisés, mis en avant, tandis que les personnages sont anonymes et au service de ces espaces.

Fig. 1 \& 2 : première image du générique ; l'escalier des serviteurs dans le générique

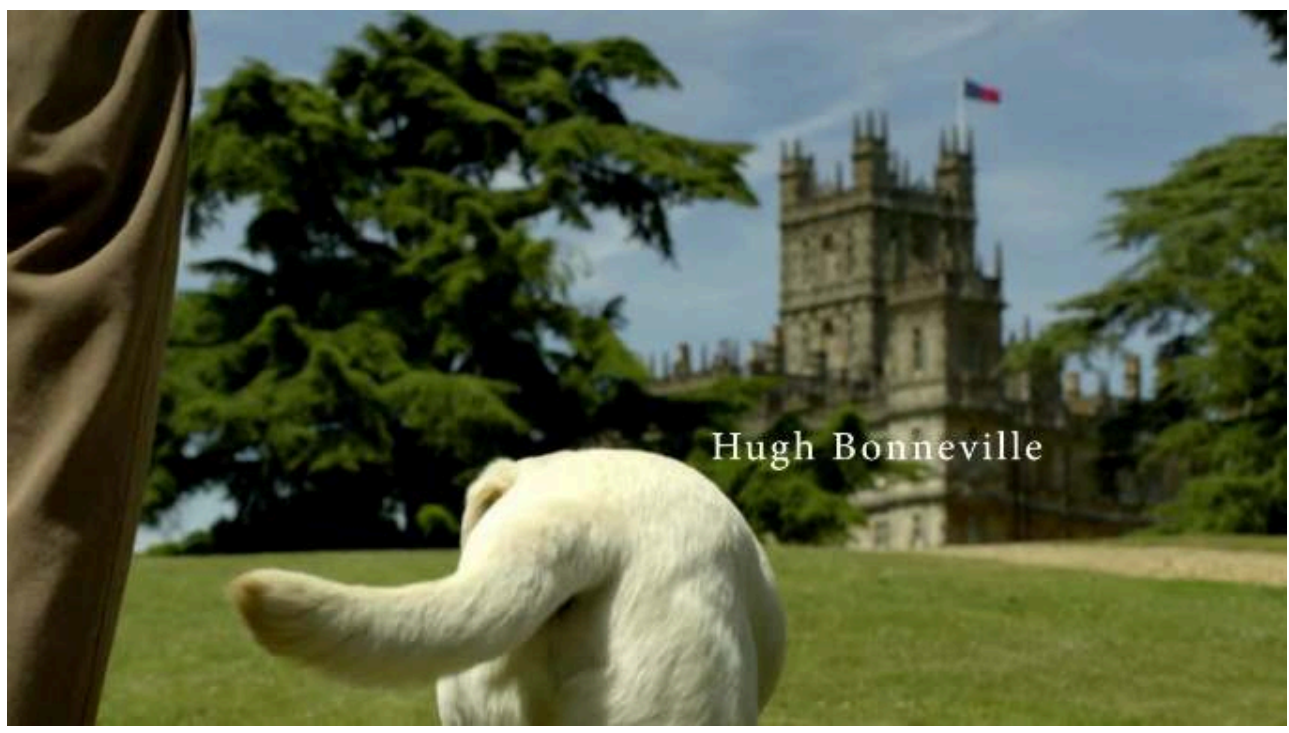




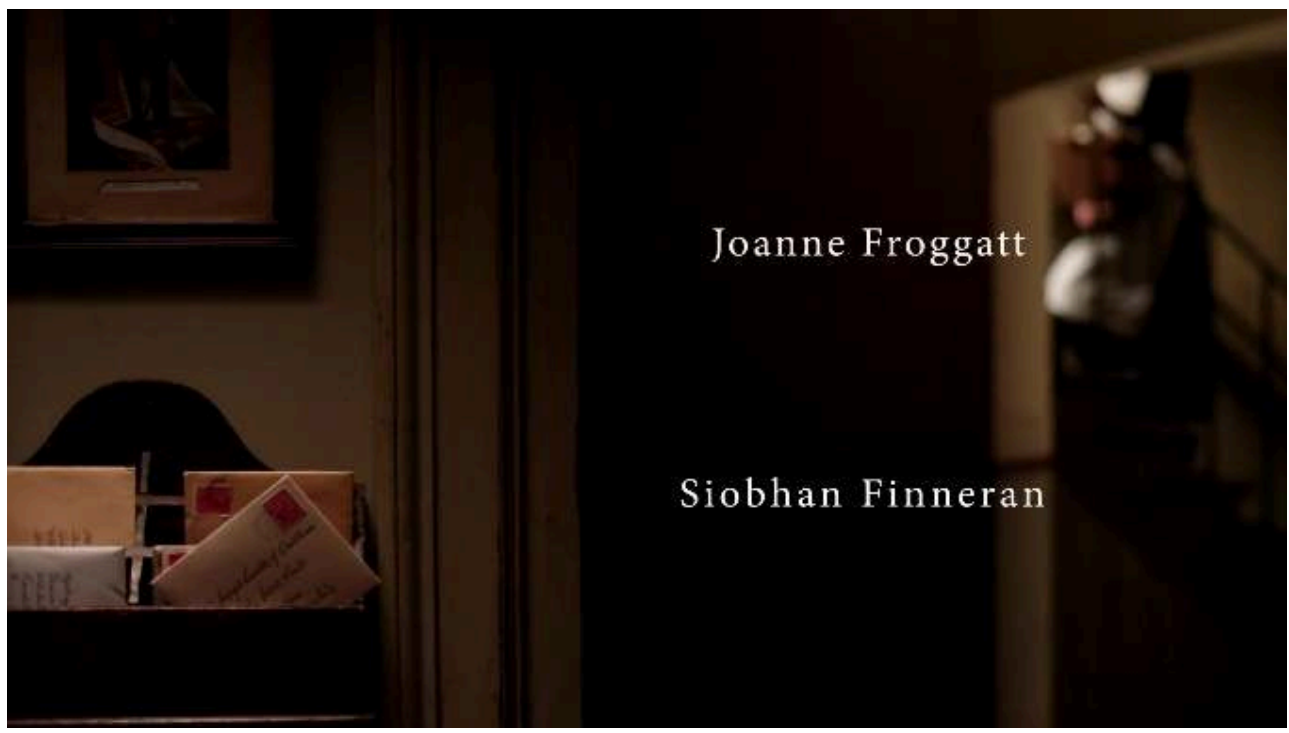

Fig. 3 \& 4 : la préparation de la table dans le générique ; le nettoyage des lustres dans le générique
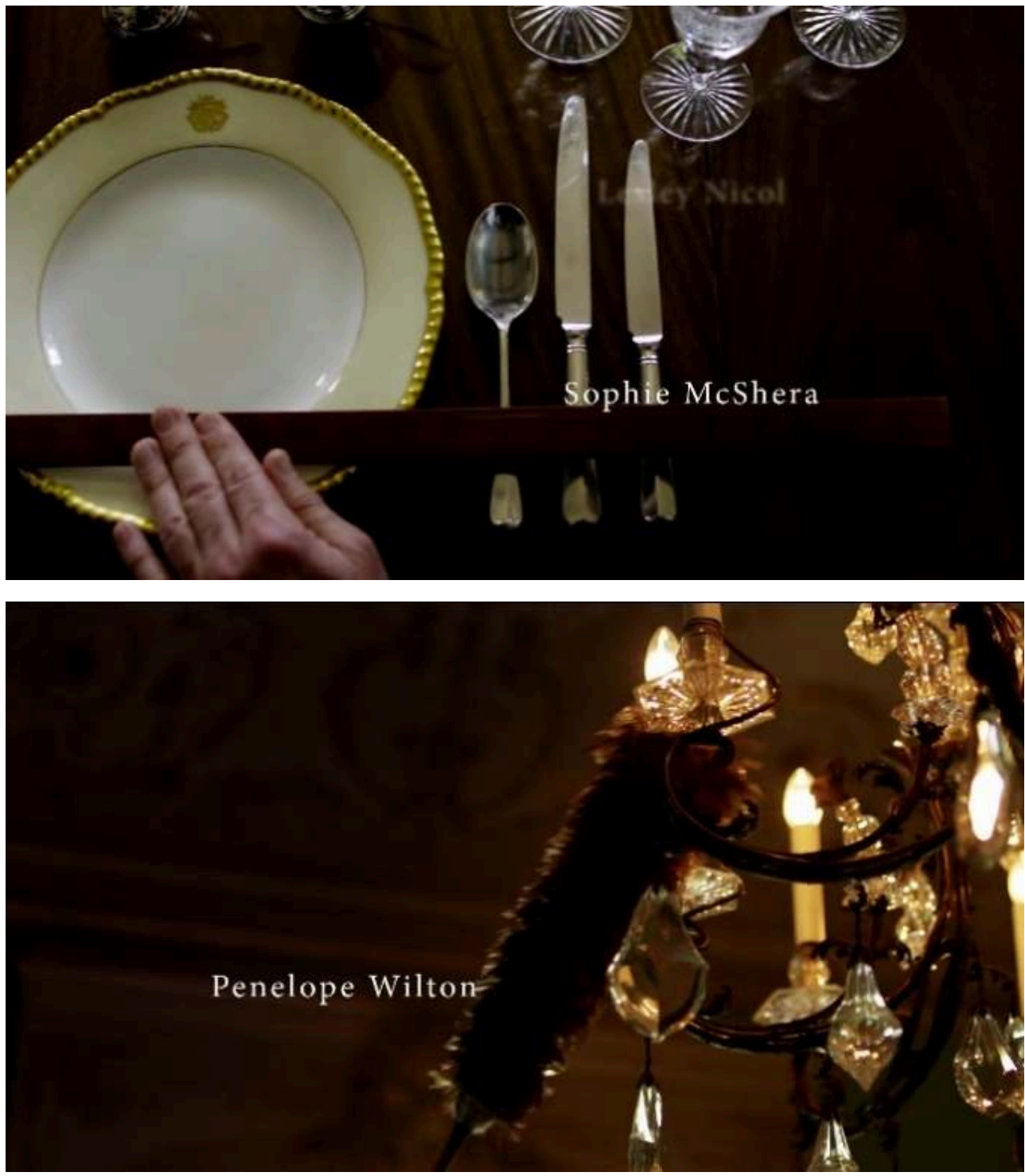
7 Le générique de Downton Abbey fonctionne comme un générique d'ambiance ${ }^{18}$, sa suite de plans sophistiqués, mettant en avant les espaces de la série, plutôt que les personnages, indique que ce sera la vie aristocratique dans ce manoir qui sera au cœur de la série. Il introduit le sujet, et son immuabilité permet de présenter un univers familier, Downton et ses différents lieux emblématiques, au spectateur au fil des épisodes puis des saisons. Les rôles sont au contraire interchangeables dans ce générique, les personnages sont renvoyés à de simples silhouettes floues et à des actions communes et quotidiennes. Au fil de la série, plusieurs personnages accompliront toutes ces actions et elles ne sont donc pas spécifiques à un rôle ou acteur précis. Cela facilite et perturbe l'identification, l'action est associée à un archétype : le majordome, une femme de chambre ou encore un valet, et non à un personnage, nommé et précis. Les changements de casting ne sont, alors, plus un problème ${ }^{19}$. Cette forme de générique peut suivre les arrivées et départs de la série, il suffit de changer les noms lors du générique, celui-ci reste immuable : la série parle de Downton Abbey, et ce sont donc ses espaces qui sont représentés pour lancer chaque épisode. La seule silhouette qui semble davantage précisée est ainsi la figure canine (Figure 1). Mais même Isis, la Labrador Retriever de la famille, quitte la série (elle meurt dans la saison 5) et est remplacée par un nouveau chien : Tiâa, de la même race.

8 La gestion de la maison est au cœur de l'intrigue, il faut préserver l'héritage et le domaine, et les garder dans la famille proche. Robert (Hugh Bonneville) qui n'a pas de fils pourra faire hériter sa fortune à ses filles, mais ne peut leur transmettre ses titres et son domaine, de plus le père de Robert a lié la fortune de Cora au domaine, les filles seront donc complètement déshéritées. Tout l'enjeu des premières saisons est ainsi de parvenir à organiser un mariage entre Mary (Michelle Dockery), l'aînée, et le nouvel héritier, pour que le domaine familial, et la fortune désormais attachée à celui-ci, restent dans la famille proche. La troisième saison se concentre ensuite sur les problèmes financiers de la famille ${ }^{20}$ et le probable abandon de Downton à cause de cela, et ce sont tous les personnages qui se retrouvent impactés. Ceux qui se montrent les plus distants de cette question sont les plus fraîchement arrivés: Cora (Elizabeth McGovern), l'héritière américaine, qui a permis par son mariage avec Robert de sauver le domaine Downton, qui accepte l'idée de déménager dans une maison plus petite ; ou encore Matthew (Dan Stevens), cousin éloigné de la famille, avocat (donc de la middle class) et nouvel héritier, qui explique dès le début de la saison vouloir revenir à une vie plus simple. Matthew est le dernier arrivé, le dernier intégré au cercle restreint de la famille, il n'entretient pas le même rapport à cet espace familial que les autres personnages de la série.

9 Mais la maison se trouve également impactée très largement par le destin des personnages qui l'habitent, notamment autour des questions de pertes et de deuil. Ainsi le premier épisode de la quatrième saison est emblématique de cette question: le personnage de Matthew Crawley est décédé dans un accident de voiture à la toute fin de la saison précédente, les téléspectateurs, tout comme les personnages, s'engagent dans cette nouvelle saison encore meurtris par ce décès prématuré, et c'est la maison qui permet pleinement d'exprimer cela (Figure 5). Dès les premières minutes de l'épisode, la maison est plongée dans le noir, on voit une silhouette qui se prépare et quitte la maison discrètement - on apprend plus tard qu'il s'agit de Sarah O'Brien, la femme de chambre de Cora - enfin, un plan montre la demeure à travers le brouillard, derrière des arbres qui obscurcissent le premier plan et resserrent le point de vue. Tout 
de suite après, on voit les différents personnages humains de la série en tenue de deuil, exactement comme Downton elle-même qui s'associe au malheur de ses occupants et paraît, elle aussi, endeuillée. À la fin de l'épisode, Mary, la veuve de Matthew, demande conseil à Carson (Jim Carter), le majordome, au fil de la discussion il lui demande de "revenir parmi les vivants ", et cela est matérialisé dès la scène suivante avec la maison qui s'illumine à nouveau, malgré le ciel encore sombre, et le fleurissement de fleurs au premier plan (Figure 6).

Fig 5 \& 6 : Downton endeuillée ; Downton surmontant son chagrin
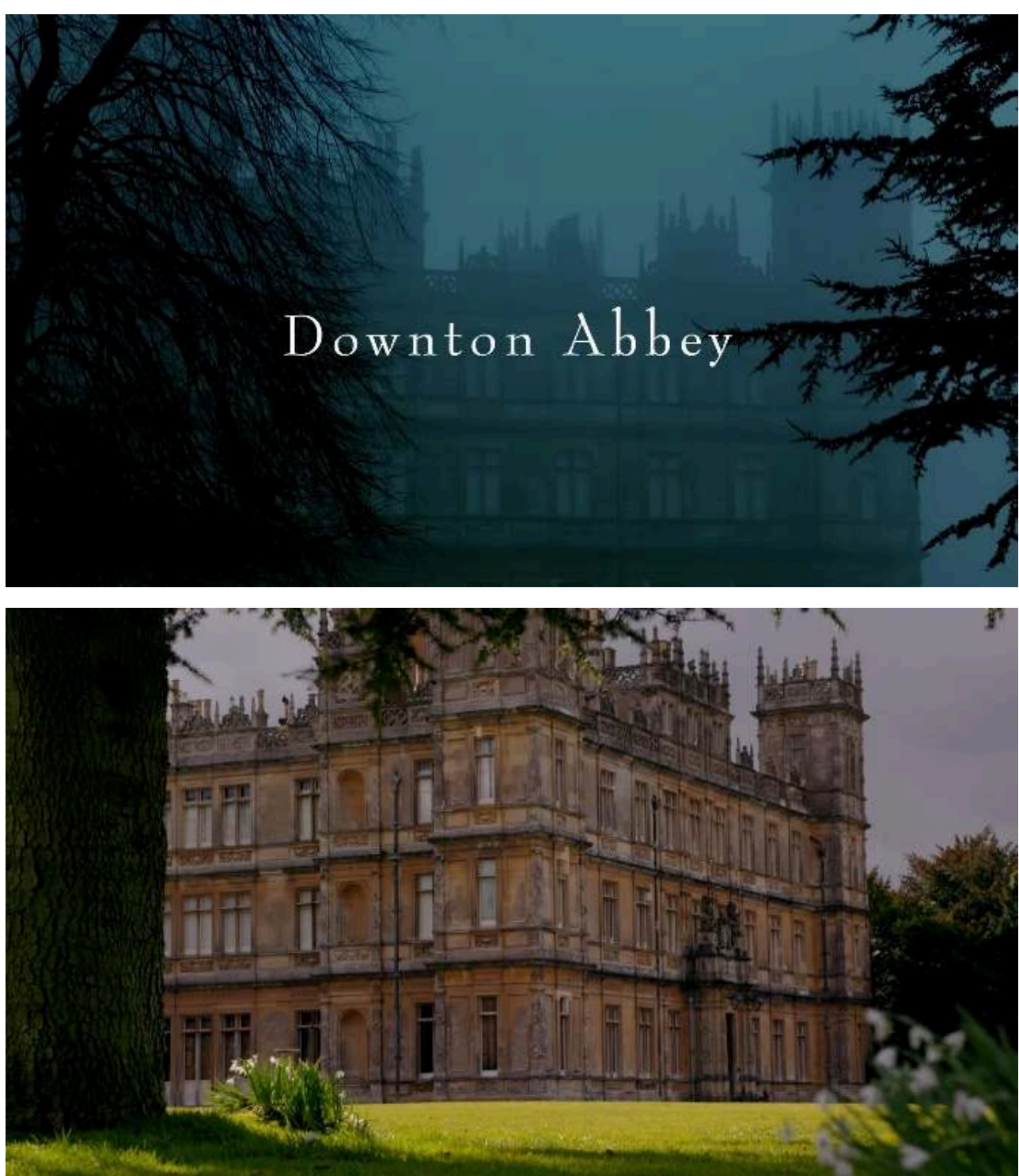

10 À l'instar des autres personnages et des spectateurs, Downton fait le deuil de Matthew et ressent de la tristesse. Mais Matthew n'est pas le seul personnage que regrette Downton, en effet lors de l'incendie déclenché par inadvertance par Edith Crawley (Laura Carmichael) (S01E05) on peut également trouver cette question du deuil. Cette dernière s'endort après avoir jeté un livre contre un mur, malencontreusement ce livre se retrouve près du foyer de la cheminée et finit par mettre le feu à la chambre. Ce livre appartient à Michael Gregson (Charles Edwards), son fiancé disparu, puis découvert mort, en Allemagne alors qu'il entamait les procédures pour leur mariage. Elle le pleure 
ainsi depuis 7 épisodes et a déjà accouché en secret. Une analyse possible de cet incendie peut être le franchissement d'une nouvelle étape dans le deuil d'Edith : l'étape quatre, celle de la dépression, de la douleur et de l'expression du chagrin et du deuil qui mène ensuite à l'étape cinq ${ }^{21}$, la terminaison du travail du deuil et la reconstruction, nécessaire, tant pour la chambre que pour son occupante. Edith souffre moins suite à cet incident, et reprend sa vie en main, tandis que la reconstruction de la chambre n'est pas montrée à l'écran (mais a bien lieu). Les émotions ne sont pas propres à Downton, elles sont parfois la reproduction de celles ressenties par ses habitants, et ce fonctionnement en miroir mérite également un développement.

\section{Fonctionnant en miroir et reproduisant la hiérarchie sociale}

11 Downton reproduit les émotions des autres personnages, elle est un reflet de ce que ses habitants vivent, mais ils se reflètent également à travers ce lieu. La maison reproduit la hiérarchie sociale et organise sa société d'habitants pour que la plupart des personnages aient un double à l'étage opposé. On peut voir cela dès l'image qui accompagne et clôt le générique pour lancer chaque épisode (Figure 7), elle résume à elle seule le fonctionnement de la maison.

Fig. 7 : image titre

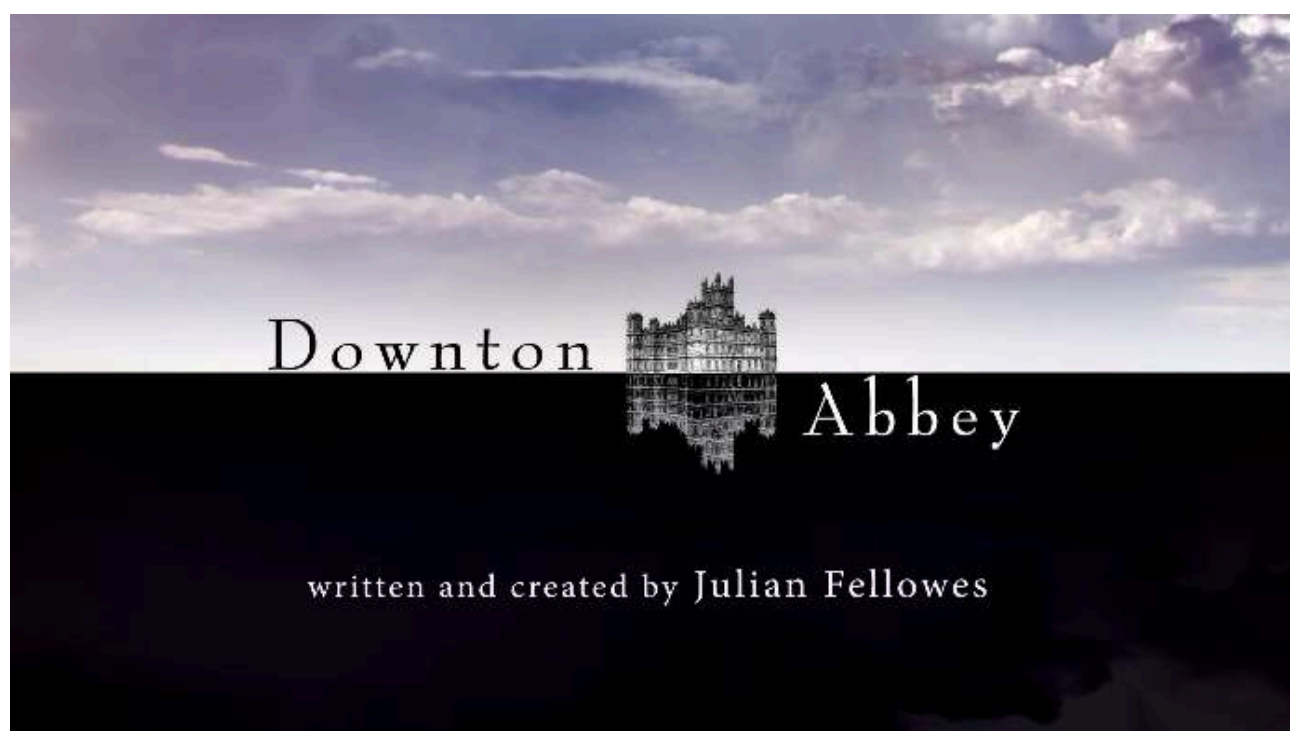

Comme dans le genre du Upstairs \& Downstairs, cette image permet de voir comment, d'un étage à l'autre, certains personnages se reflètent dans d'autres, quel que soit leur milieu d'origine. Tout d'abord l'intransigeance morale se retrouve chez John Bates, le valet, et Robert Crawley, le chef de famille : ils ont servi ensemble dans la guerre des Boers, et sont souvent préservés de nombreux problèmes par leur femme ${ }^{22}$. Mais on peut également voir cette proximité avec les personnages de Gwen (Rose Leslie), une des femmes de chambre, et Sybil Crawley (Jessica Brown Findlay), la benjamine de la famille. Gwen, qui a pris conscience des rapports de domination, souhaite s'émanciper et quitter la maison pour trouver un autre travail et ne pas être domestique toute sa vie, et c'est Sybil qui l'aide à accomplir son rêve (Saison 1) et qui réalisera la même démarche émancipatrice pour elle-même lors de la saison suivante. Elle devient infirmière pour ne pas se contenter de rester à Downton en temps de guerre et profite 
du climat de changement et de possibilités pour se mettre à travailler. Cette dynamique se retrouve après le départ de Gwen (S01E07) à travers le couple que forment Tom Branson, le chauffeur irlandais, et Sybil. Tous deux portent en eux une fibre révolutionnaire et un désir d'émancipation, tant politique que sociale, qu'ils tenteront de pousser à leur maximum, allant jusqu'à quitter, temporairement, cet espace qu'ils estiment conservateur et réfractaire au changement. On peut également retrouver dans la relation entre Mary et Edith Crawley, la cadette de la famille qui entretient des relations houleuses avec sa grande sœur, de grandes similitudes avec la relation entretenue par Sarah O'Brien (Siobhan Finneran), la femme de chambre attitrée de Cora, et Thomas Barrow (Rob James-Collier), un des valets de pied. En cherchant toujours un moyen de jouer un mauvais tour à l'autre, les deux sœurs Crawley occupent une partie importante de l'intrigue, tandis que les machinations de Barrow et O'Brien sont également un des moteurs principaux des évènements chez les domestiques. Les personnages et les relations se lient, se répondent et se complètent d'un étage à l'autre.

Comme le suggèrent le blanc et le noir présents dans l'image (Figure 7), la série pose également des questions morales et joue à inverser les rôles. Ce sont les personnages de l'espace inférieur, du downstairs, pourtant symbolisé par le noir, qui sont le plus souvent garants de l'ordre moral. Bates que nous avons déjà évoqué, mais également Carson, et plus que tout, Anna (Joanne Froggatt), à la tête des femmes de chambre, qui au fur et à mesure des saisons est de plus en plus un miroir pour Mary, telle qu'elle devrait être. L'exemple le plus marquant de cela est sans nul doute lorsque Mary organise sa semaine avec Lord Gillingham (S05E02), un de ses prétendants, elle en discute avec Anna, expliquant son point de vue: il faut connaitre la personne, y compris sexuellement, avec qui on envisage de passer sa vie. Anna lui répond qu'elle est trop «vieux jeu» pour cela. Mary demande ensuite à Anna d'aller acheter un contraceptif, livre de Marie Stopes ${ }^{23}$ à l'appui, pour organiser sa semaine. Anna qui est véritablement l'incarnation de la tradition et de la bonne conduite refuse tout d'abord cette demande avant d'y accéder à contrecœur. C'est bien aux personnages du downstairs que revient la tâche impropre, mais pour le compte des personnages upstairs qui restent "propres» de ces activités ${ }^{24}$. Tout comme dans l'image titre, Downton repose sur une opposition entre les espaces, et entre ses personnages.

Mais elle est aussi un miroir de l'époque représentée, et le rapport à ses espaces se reproduit parmi le casting. L'arrivée de Matthew dans la série (S01E02) et à Downton est ainsi marquante pour cela. Pour Katherine Byrne, Matthew fonctionne comme un représentant de la classe moyenne urbaine ${ }^{25}$, qui serait le public ciblé par la série. Son arrivée à Downton reproduirait alors la sensation des téléspectateurs arrivant dans ce monde de l'aristocratie britannique et des country houses lors du premier épisode : un mélange de stupeur, d'admiration et de distance, voire d'écœurement et de dégoût face au mode de vie qu'entretient l'aristocratie. On peut largement nuancer cette vision, ou tout du moins la compléter; plusieurs séries ou films se déroulent dans des univers similaires et ont fixé certains codes et certaines représentations pour le téléspectateur ${ }^{26}$. Toutefois, Downton Abbey est la première série d'envergure depuis dix ans à s'illustrer dans ce genre, aussi le spectateur générique dont parle Katherine Byrne est plutôt un jeune téléspectateur, qui découvre le upstairs downstairs avec Downton Abbey. Mais au-delà du personnage de Matthew et de sa symbolique pour faciliter l'introduction d'un certain type de téléspectateur à ce monde qu'il ne connaît pas, cela permet également de voir naître dans ce personnage un sentiment d'appartenance à ce monde, sa prise de responsabilité jointe et son attachement, que peut également 
ressentir le spectateur. Le personnage de Tom Branson suit exactement le même schéma narratif, cette fois-ci pour les classes ouvrières pauvres. Lui qui était révolutionnaire et indépendantiste prend également conscience de l'importance de Downton et change d'opinions et d'idées politiques. Il adoucit ses revendications et opinions : lui qui était fermement opposé à la domination sociale et l'organisation de la société en classe, finit par vivre à Downton et à s'illustrer en pérennisant cet ordre établi.

Cette hiérarchie sociale et son fonctionnement se retrouvent au sein du casting. Dans les différents livres making-of de la série, on peut ainsi apprendre que le conseiller historique, Alistair Bruce, a ainsi maintenu une division entre les acteurs "endessous» (below) et "au-dessus» (above) sur le tournage, pour leur expliquer ce qu'aurait été la vie pour eux à cette époque. Cette division entre acteurs se manifeste également par l'accès aux espaces. Pour la saison 1 notamment, les scènes dans les chambres ou au rez-de-chaussée étaient réellement tournées au château de Highclere, tandis que les espaces inférieurs, la salle des serviteurs et les différents autres lieux domestiques étaient construits dans les Studios Ealing ${ }^{27}$. Une partie des acteurs jouant des domestiques tournait donc exclusivement en studio. Lors de scènes exceptionnelles, comme la discussion entre Mrs Patmore et Robert Crawley dans la bibliothèque, Lesley Nicol l'actrice incarnant la cuisinière, a ressenti également cet émerveillement, ce sentiment de ne pas être à sa place que pouvait ressentir une cuisinière du début du $\mathrm{XX}^{\mathrm{e}}$ siècle ${ }^{28}$.

Ce sentiment d'appartenance au lieu, à l'espace de vie, est également au cœur de la réflexion et du travail de Penny Dyer, la conseillère dialogue de la série. Pour elle, la différence entre la façon de parler entre les acteurs above et les acteurs below ${ }^{29}$ n'est pas une question de prononciation des mots, mais davantage une façon de porter la voix. Pour elle toujours, ceux qui ont grandi dans des maisons petites, encombrées et avec de grandes familles auraient la voix portant sur les côtés, ne se projetant pas et tentant de se frayer un espace, tandis que les aristocrates projetteraient naturellement leurs voix pour remplir des pièces plus grandes ${ }^{30}$. De plus, il est également au cœur du travail de réalisation, notamment de Brian Percival, le réalisateur de plusieurs épisodes ${ }^{31}$. Il explique en effet que pour représenter deux états d'esprit différents : above qui serait calme et serein et pour lequel il a utilisé des plans larges et de longs travellings, et below qui serait plus naturel, énergique, et pour lequel il a fait le choix d'utiliser des caméras portées et des plans à l'épaule pour donner un rendu plus naturel, presque documentaire ${ }^{32}$. Les lieux, et leurs positions upstairs ou downstairs dans la maison sont donc au cœur du travail de réalisation. Le style d'image et la mise en scène permettent de refléter des états d'esprit différents, mais également et principalement, des positions sociales. L'appartenance aux lieux et aux espaces est centrale pour les personnages. La façon de les filmer et de les caractériser lors d'une scène ne dépend alors pas de leur propre personnalité, mais de l'ambiance et de l'état d'esprit du lieu dans lequel ils se trouvent. Les différents espaces de la maison définissent le caractère et le tempérament des scènes. 


\section{Une demeure conservatrice}

\section{Une maison-nation qui protège...}

17 Katherine Byrne propose de voir dans les personnages de Downton Abbey un microcosme représentant un État, une nation ${ }^{33}$. C'est en partant de cette idée de Downton comme une allégorie étatique que se poursuivra notre développement. On peut porter crédit à cette analyse, l'espace de l'histoire est uniquement Downton et plus généralement le domaine Downton. La maison fonctionne comme une synecdoque du Royaume-Uni et tente de décrire un mode de vie idéal qui repose sur une hiérarchie de classe et une charité paternaliste. Dans la maison lorsque tout le monde est à sa place et accomplit sa mission, le domaine prospère. Cette mise en scène des rouages nécessaires au bon fonctionnement d'une maison - d'une nation - relève d'une approche conservatrice, nostalgique, et s'intègre pleinement dans le genre heritage.

Downton n'a pas de système de sécurité sociale, elle n'en a pas besoin, et c'est la figure paternaliste, incarnée par le patriarche Robert, qui prend en charge ce système. Il s'occupe ainsi de la réinsertion d'anciens combattants, de l'emploi de travailleurs handicapés, ou même tout simplement les frais médicaux afin d'épargner une perte de travail. Lorsque Mrs Patmore commence à perdre la vue, c'est Lord Grantham qui prend en charge les frais de son opération de la cataracte (S01E07), c'est également Lord Grantham qui donne une chance à Bates en tant que domestique, et ce malgré sa blessure à la jambe, son boitement et sa nécessité d'utiliser une canne (S01E01). C'est également lui qui, contre toute attente, s'oppose à la décision de Carson de le renvoyer, car il ne peut pas accomplir pleinement son travail (S01E01 également). L'initiative sociale est privée, elle repose sur la charité et non sur le gouvernement. En effet, à Robert s'ajoute également sa fille, Mary, qui prend en charge les difficultés de grossesse d'Anna à plusieurs reprises dans la saison 6, en s'occupant des frais et des démarches. Ou encore ce que rappelle Katherine Byrne : c'est Mrs Hughes qui, lors de la saison 3, craint un cancer depuis plusieurs mois et consulte en cachette s'avère ne pas être malade le jour de sa discussion avec $\operatorname{Cora}^{34}$ qui la réconforte et lui annonce que la famille fera tout le nécessaire pour pouvoir s'occuper d'elle en cas de diagnostic négatif (S03E03). Le système d'assurance maladie de Downton fonctionne selon un modèle patriarcal qui repose sur la charité du patriarche et de la famille. Ce dernier permet alors de mettre à l'abri tous les membres, à condition de rester dans l'enceinte familiale.

Mais cette protection ne s'arrête pas à une simple aide médicale, cela va plus loin. Lorsque Mr Mason, le désormais beau-père de Daisy (Sophie McShera), la fille à tout faire puis l'aide-cuisinière, va être expulsé de la parcelle qu'il occupe depuis plusieurs décennies dans un autre domaine, les gérants du domaine Downton, que sont Mary Crawley et Tom Branson, trouvent une solution. Ils lui proposent alors de reprendre une des exploitations du domaine. Lorsque l'on est lié de près ou, et dans ce cas-ci, de loin à la demeure, on bénéficie de sa protection quotidienne, même professionnellement. Lorsque Carson est menacé par un ancien ami qui tente de le soumettre à un chantage, c'est Lord Grantham, une nouvelle fois, qui le protège et paye l'homme en l'obligeant à promettre de ne jamais contacter Carson à nouveau (S01E02). Il réitère cet exploit, lorsque Mary est menacée à son tour par une tentative similaire, qui dévoilerait son aventure amoureuse avec Lord Gillingham (S06E01). Cette 
protection concerne donc, également, des questions de mœurs, le système paternaliste protège les membres de la famille. Ainsi Ethel (Amy Nuttall), une femme de chambre qui a une aventure avec un soldat en convalescence à Downton, tombe enceinte et qui se voit contrainte de quitter le service, fait ensuite « de mauvais choix de vie ${ }^{35}$ ». Elle est aidée tout d'abord par Mrs Hughes (Phyllis Logan), intendante et à la tête des femmes de chambre, qui lui donne à manger et tente d'établir un plan pour rencontrer les grands-parents de l'enfant, puis par Isobel Crawley (Penelope Wilton), la mère de Matthew, qui avec l'aide de Mrs Hughes parvient à donner un meilleur futur à l'enfant en le confiant à la garde de ses grands-parents. Isobel Crawley poursuit son aide, et engage Ethel comme cuisinière, et ce malgré les vives oppositions de Mrs Bird sa cuisinière. Thomas est également protégé par la famille. Alors que son homosexualité a été dénoncée par Jimmy, un des valets de pied, Carson tente d'étouffer l'affaire et d'empêcher Jimmy d'en parler à la police, sur conseil de lord Grantham. Puis, alors que la police a finalement été prévenue, par Alfred, un autre valet de pied, et que deux inspecteurs viennent lors du match de cricket pour arrêter Thomas, c'est Lord Grantham à nouveau qui convainc Alfred de se rétracter sur sa déposition (S03E08). Downton apparaît donc, malgré ses divers aspects nostalgiques, comme une nation alternative au Royaume-Uni du début $\mathrm{du} \mathrm{XX}^{\mathrm{e}}$ siècle. Elle protège tous ses membres, contre le véritable monde, plus dur, plus froid, plus hostile. C'est une nation autonome, avec des références explicites à ce que devrait être l'ordre social, mais elle tente également de se montrer progressiste et très largement anachronique sur les questions de morale. La nostalgie et le sentiment conservateur de Julian Fellowes ne parviennent pas à éloigner totalement Downton Abbey du genre de l'heritage et de la vision idéalisée du Royaume-Uni du début du siècle qu'il entretient.

Mais cette protection va également dans les deux sens, et les domestiques contribuent très efficacement au fonctionnement de ce cocon protecteur. Mary et sa réputation sont très largement protégées par le microcosme familial de Downton. Ainsi, lors de l'aventure avec Kemal Pamuk, le fils de l'ambassadeur de Turquie en visite chez les Crawley, qui séduit Mary dans la nuit avant de finalement mourir dans son lit, c'est Anna et Cora, une femme d'en haut et une d'en bas, qui aident Mary à dissimuler la cause de la mort. Bates s'illustrera aussi à plusieurs reprises, préférant céder au chantage de sa femme, lui donner de l'argent et rester marié avec elle, pour protéger la réputation de Mary ${ }^{36}$. Lorsque William se sacrifie sur le front pour tenter de protéger Matthew on peut voir une nouvelle illustration de cette protection de l'aristocratie par ses domestiques dévoués. Les fidélités entre domestiques et la famille perdurent même en dehors de l'espace Downton. On peut également mettre en avant le souhait de Carson: protéger la «nation» en préservant des standards de service, pour lui cela permettra de montrer aux Allemands qu'ils ne gagneront pas la guerre (S02E01 et E02). Pour cela il effectue alors le travail de plusieurs domestiques tout seul, ce qui le mène à s'effondrer de fatigue.

Il n'existe aucun problème qui en peut être dépassé grâce à de l'amour, de la loyauté, et un esprit de corps au sein de la maison-nation Downton. Lorsque ce n'est plus le cas, et qu'un doute intervient dans une relation, par exemple celle de Cora avec sa femme de chambre Sarah O'Brien, des conséquences dramatiques sont à déplorer ${ }^{37}$. Tout cela relève véritablement d'une vision conservatrice : présenter une famille aristocratique qui défend et protège ses domestiques, tellement aimante et digne d'amour que les domestiques en font de même, relève d'un fantasme de la country house $e^{38}$, et de la 
société en général. Downton est le lieu de tous ces miracles et de cette vision déformée et nostalgique de l'aristocratie et de l'Angleterre du début du XX ${ }^{e}$ siècle.

\section{... et met à l'écart}

Dans Downton Abbey, le monde extérieur n'existe pratiquement pas. Le contexte mondial est évoqué dans ses grandes lignes : naufrage du Titanic (S01E01), assassinat de François-Ferdinand (S01E07), entrée en guerre du Royaume-Uni (S01E07), Première Guerre mondiale (Saison 2), Révolution d'octobre (S02E03), fin de la guerre (S02E06), épidémie de grippe espagnole (S02E08). Mais hormis ces quelques évènements majeurs, et incontournables pour permettre aux téléspectateurs de situer l'action, le contexte social et politique du Royaume-Uni est très largement mis à l'écart du récit. L'image qu'une nation a d'elle-même est fortement impactée par ses productions culturelles. Benedict Anderson explique ainsi qu'une nation n'existe que comme construction, et c'est en s'adressant à une "communauté imaginaire", notamment par le biais d'œuvres culturelles, qu'elle est créée ${ }^{39}$. Il convient alors d'analyser le récit historique du début du XXe siècle produit par Downton Abbey.

Bien que l'entrée en guerre du Royaume-Uni clôture la première saison, la guerre est mise à l'écart de la maison, de la famille. On ne perçoit que le discours de l'élite ou les collectes de fonds, mais l'impact profond de la Guerre, sur les mentalités, sur la hiérarchie sociale, sur l'aristocratie britannique, est complètement évacué. Au sein du microcosme Downton, la guerre est concentrée en quelques points, et épurée, bien loin de la profonde déflagration qu'elle a produite et de la mortalité qu'elle a engendrée dans l'aristocratie. La blessure et le geste de Thomas, qui provoque sa propre mutilation sur le front afin d'échapper aux tranchées, sont vite évacués, et sont complètement occultés dans les saisons suivantes. La comtesse douairière tente de protéger la plupart des hommes liés à la famille, elle trouve des explications et parvient à empêcher l'enrôlement de Molesley (Kevin Doyle), le valet de Matthew, et, dans un premier temps, celui de William (Thomas Howes), un valet de pied de Downton. William rejoint finalement le front et meurt après avoir tenté de protéger Matthew. Ce dernier revient du conflit blessé, mais finit par remarcher miraculeusement. Ce n'est pas la guerre, mais des contraintes scénaristiques - l'acteur souhaitant quitter la série ${ }^{40}-$ qui auront raison de lui, Matthew mourant ainsi dans un accident de voiture lors de la dernière scène de la troisième saison. Downton Abbey tente pourtant de traiter les séquelles de la guerre. Tout comme de nombreuses country houses Downton est partiellement aménagée comme un hôpital ${ }^{41}$, cela est également une réalité historique du château de Highclere pendant la Première Guerre mondiale ${ }^{42}$. Mais les conséquences sur la vie des soldats, sur la vie des blessés, sont totalement absentes. Ce qui est représenté dans la série, ce sont les conséquences de cet aménagement, de cette cohabitation pour les membres de la famille. On retrouve également ce traitement avec le personnage de Lang, un soldat traumatisé et démobilisé. Il est le seul personnage de la maison qui souffre véritablement de la guerre, le problème est complètement épuré puisque concentré en un seul personnage. De plus, ce seul personnage incarnant le traumatisme de la guerre est évacué de l'histoire et de la maison en un seul épisode. Malgré le soutien, très contemporain dans sa philosophie, de Lord Grantham pour Lang - qui aurait plutôt dû voir en ce traumatisme de la lâcheté ou de la folie ${ }^{43}-$ il éloigne quand même les conséquences de la guerre de la maison. 
Les relations conflictuelles avec le nord du Royaume et avec l'Irlande sont, à la manière de la Première Guerre mondiale, épurée et réduite à un seul personnage : Tom Branson. Lui qui défend l'indépendance de l'Irlande et l'IRA est bien pacifiste. De plus, les luttes armées entre unionistes et républicains en Irlande sont évacuées de la série, et même très largement minimisées, la reconnaissance de l'Irlande du Nord est également complètement absente. Tom désavoue seulement l'incendie de la maison de ceux qu'il considère comme des oppresseurs de l'Irlande (S03E04). L'acte de terrorisme que prépare ce "révolutionnaire » est également complètement pacifié puisqu'il consiste en un renversement d'une mixture ressemblant à de la poix sur un général en visite à Downton. L'aspect révolutionnaire des personnages est complètement vampirisé par la maison et disparait très vite de leur caractère. Ainsi, Branson qui avait probablement la conscience socialiste la plus poussée de la série est progressivement adouci, à tel point qu'il ne se reconnaît plus (S04E03), il finit même par admettre que toutes ses idées politiques se sont envolées (S04E07). Ce sont des personnages extérieurs à la demeure qui doivent les lui rappeler (Isobel Crawley ou Sarah Bunting). Mais dans le cas de Sarah Bunting (Daisy Lewis), une institutrice du village sensible aux idéaux socialistes, on lui fait très rapidement remarquer que ses idées n'ont pas leur place dans ce milieu. Cette mise en garde se fera principalement par Robert Crawley qui au terme d'une nouvelle confrontation avec elle, lui dit de quitter la maison et qu'elle n'est pas la bienvenue. Au cours de cette discussion à la fois la mise en scène et les dialogues ne permettent aucune identification du spectateur avec elle, comme le rappelle très justement Laetita Kevers $^{44}$. La scène est filmée depuis le point de vue de l'aristocratie et Sarah est uniquement présentée pendant ses arguments, jamais en réaction, ce qui ne permet aucune empathie. Lors de cette discussion, Violet lui fait également remarquer que «les principes sont comme les prières: nobles, mais déplacés à une soirée » (S05E01). Un peu plus tôt dans la série elle est même rappelée à l'ordre par un domestique, Thomas, lorsque ce dernier la trouve en compagnie de Tom à l'étage des chambres. C'est son insubordination, sa transgression, sa présence upstairs qui est une anomalie, une transgression. Aussi, très vite elle redescend pendant que Thomas les regarde du haut de la mezzanine (Figure 8) sur fond du thème musical de la trahison et de la manigance ${ }^{45}$. Sarah quitte la série et ce milieu social (S05E05), ses idéaux socialistes étant trop affirmés et intransigeants ils n'ont pu être dissous, réfrénés ou contenus ; aussi malgré les différents rappels à l'ordre, elle est écartée de l'histoire et éloignée de la demeure. 
Figure 8 : Thomas scrutant le départ de Sarah Bunting

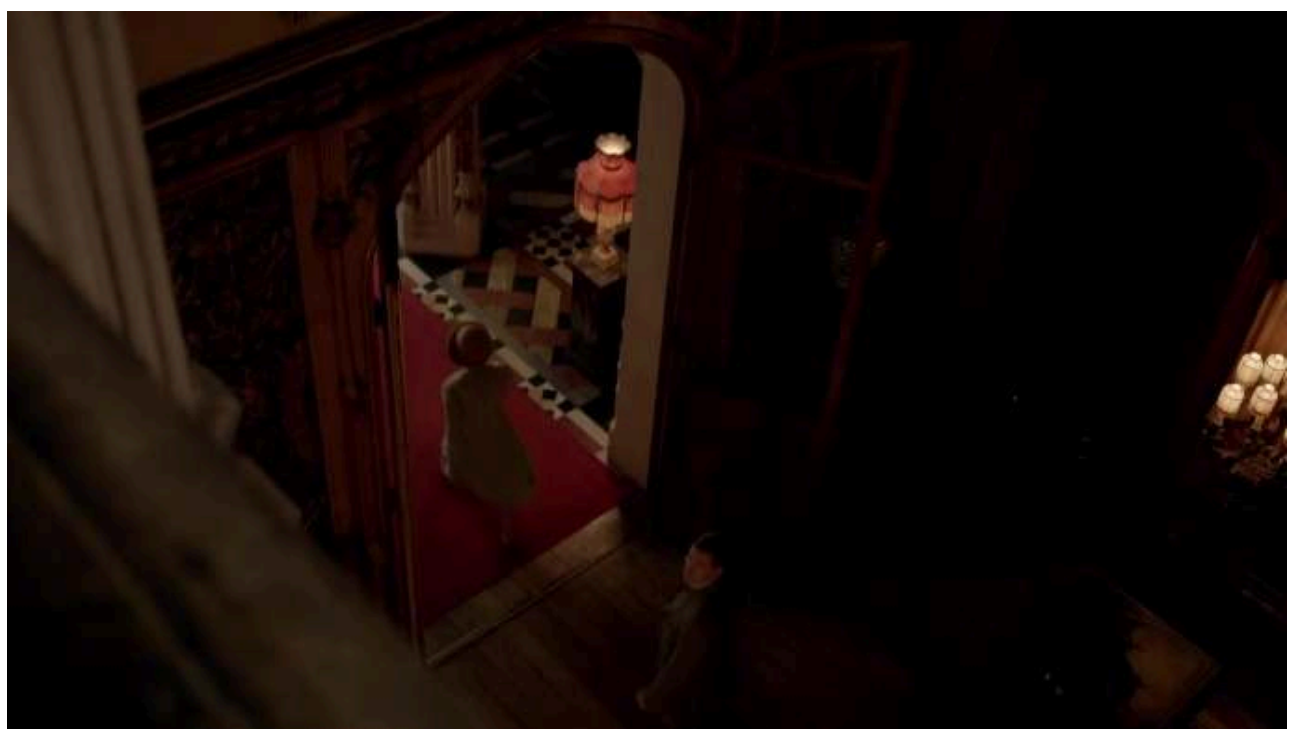

Enfin, si l'on considère à nouveau la maison comme un personnage de la série, on peut analyser la façon dont certains sujets en sont éloignés. Une analyse complémentaire de l'incendie dans la chambre d'Edith (S05E01) peut être la préservation consciente de l'ordre moral par Downton. Même si la famille est progressiste sur certains sujets, la maison se montre moins conciliante: l'ordre social ne peut être transgressé aussi facilement. Aussi, l'incendie de la maison contraint à une évacuation. Robert Crawley passe ainsi dans toutes les chambres afin de réveiller et faire sortir les occupants de la maison, ce qui lui permet de se rendre compte de l'écart de Jimmy, qu'il retrouve dans le lit de Lady Anstruther. Dès le lendemain Jimmy se voit contraint de quitter la maison, tandis que Lady Anstruther part en pleine nuit. Edna, une domestique, qui tente de séduire Tom Branson se voit également éloignée (S03E09). Downton est un espace de préservation morale et sociale, et malgré l'adoucissement de ses convictions, elle ne peut pas permettre que la hiérarchie sociale soit ainsi bafouée.

Il en va de même pour Mary, lors de son aventure avec Kemal Pamuk (S01E03). Ce dernier est le fils de l'ambassadeur de Turquie, il accompagne un ami de la famille chez les Crawley, et au milieu de la nuit il se rend dans la chambre de Mary qu'il parvient à séduire. Les conséquences de cette aventure sont doublement graves: elle nuit considérablement à la réputation de Mary, et cela conduit même à la rupture de ses fiançailles, mais elle coûte également la vie à Kemal Pamuk. Downton est bien plus violente et conservatrice que le patriarche. Toutefois, la demeure s'adoucit progressivement : l'épisode de l'incendie est ainsi également celui dans lequel Mary et Lord Gillingham concluent leur plan de devenir amants l'espace d'une semaine afin d'être sûrs de leurs sentiments avant un possible mariage; Mary cherche désormais à se préserver, et c'est à Londres qu'aura lieu cette escapade romantique. On peut remarquer progressivement que la demeure se montre plus conciliante, elle ne tue plus, elle se contente de prévenir et d'éviter les scandales.

\section{Comment s'émanciper dans cette demeure?}

Comme le rappellent Laurence De Cock, Mathilde Larrère et Guillaume Mazeau dans leur dernier essai, L'histoire comme émancipation, on peut tout à fait émanciper avec de 
grandes figures ou des personnages issus de l'aristocratie, et aliéner avec des héros populaires ${ }^{46}$. L'important est ainsi le traitement fait de ces personnages et les questions qu'ils permettent de creuser et de poser. Puisque nous considérons les fictions historiques comme une écriture de l'histoire ${ }^{47}$, la question de l'émancipation, extrêmement liée au lieu familial et aux différents espaces du Royaume, se doit d'être posée. Cette partie de notre développement prendra donc le contre-pied de l'article de Laetitia Kevers, "Re-establishing Class Privilege » dans lequel cette dernière affirme que Downton Abbey ne peut être vu comme progressiste, à plus forte raison sur la question des femmes ${ }^{48}$. Son analyse très juste sur l'écriture des personnages, notamment des classes populaires, peut être nuancée par certains aspects.

\section{La transgression de classe}

Plusieurs personnages transgressent le sentiment de classe ou l'ordre social et parviennent à exprimer une vision plus émancipatrice de la société britannique et du monde pendant les six saisons de la série. La plus évidente transgression de classe est bien entendu celle du couple composé de Tom Branson et de Sybil Crawley. Tom échange ses opinions politiques avec Sybil et tous deux se forment politiquement (Saison 1 et 2). Sybil est la première des filles de la famille à s'émanciper : dès la première saison, elle ose être avant-gardiste et porte une robe/pantalon (S01E04). Elle montre son indépendance d'esprit et d'opinions par ses tenues vestimentaires. Les membres de la famille ne s'y trompent pas et se montrent surpris, voire choqués, de la tenue (Figure 9) ${ }^{49}$. Cette forme d'émancipation, à travers un contrôle de son apparence, est également adoptée par Mary lors de la saison 5. Elle se coupe court les cheveux et porte fièrement cette nouvelle allure à la garçonne ${ }^{50}$ (Figure 10), à la fin de son rendezvous chez le coiffeur elle remercie même ce dernier, car il l'a « rendue beaucoup plus forte ». Alors qu'elle devient plus indépendante, et tente d'affirmer ses choix et d'embrasser la modernité, Mary suit le modèle de sa sœur. Elle qui reprochait à Sybil de travailler dans la deuxième saison, assume pleinement son rôle d'agent du domaine et se bat même avec son père pour imposer sa présence. 
Fig. 9 \& 10 : la nouvelle robe de Lady Sybil (S01E04) ; la nouvelle coupe de Lady Mary (S05E06)
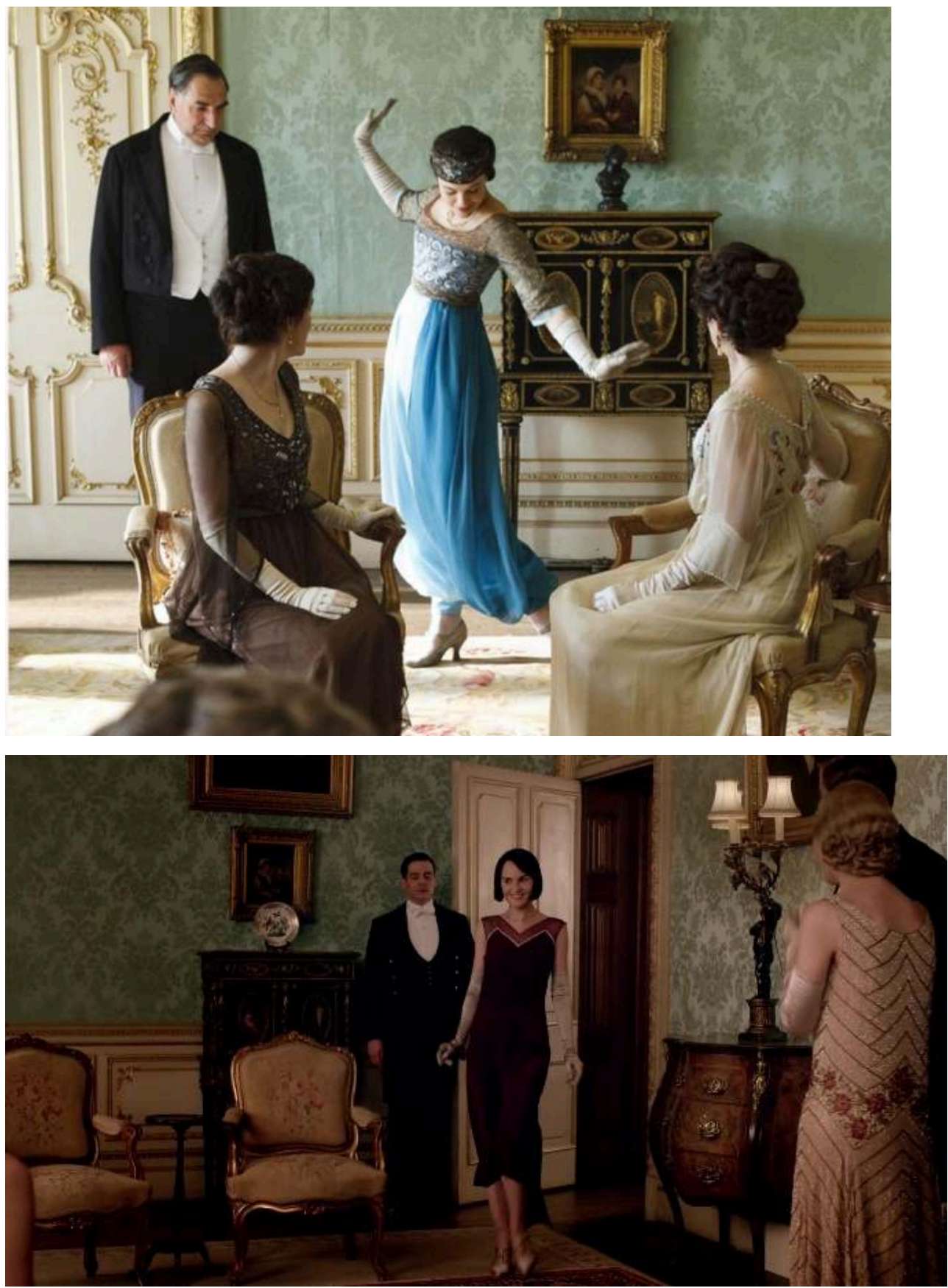

29 Sybil décide également de se former et d'apprendre un métier, lassée de sa vie oisive à Downton. Pour ce faire, elle demande notamment l'aide de Mrs Patmore et de Daisy en cuisine. L'organisation de la maison et la présence de domestiques ainsi que d'un lieu qui leur est dédié permettent à Sybil de se confronter à une réalité différente, de s'épanouir et de s'émanciper. Malgré Carson, très déboussolé par la présence de Sybil en cuisine depuis plusieurs jours, et qui va jusqu'à prévenir Cora, l'espace familial lui donne cette possibilité. Pour Tom, la transgression est plus subtile, tout d'abord on lui permet la lecture des ouvrages présents dans la bibliothèque de la famille, un des premiers pas pour former ses opinions. Les discussions avec Sybil lui permettent également de formuler ses idées, c'est à elle qu'il annonce par exemple qu'il refuse 
d'être envoyé au front et qu'il se déclare objecteur de conscience. Leur séduction n'est pour autant pas entravée et tous deux finissent par décider de leur mariage. Robert refuse dans un premier temps, avant de revenir sur sa décision et de donner sa bénédiction à Sybil. Downton permet cette rencontre et cette émancipation des deux personnages, et alors que leur histoire aurait pu être entravée par le patriarche elle est soutenue. Downton est un lien de rencontre, de transgression et de transition de classe.

Certains personnages deviennent des agents d'émancipation. C'est ainsi le cas de Sybil, qui soutient et parraine le projet de Gwen, une femme de chambre, de ne pas rester domestique et de devenir secrétaire. Toute la première saison voit ainsi plusieurs tentatives, qui finissent par se solder par une réussite: Gwen est embauchée comme secrétaire dans une société de téléphone. Pour Laetitia Kevers, cette situation n'est pas émancipatrice, car le bonheur des classes populaires découle de la bonne volonté de l'aristocratie $\mathrm{e}^{51}$. En cela, il y a une part de vérité, mais également une simplification qui ne rend pas compte de la réalité. La demeure permet à Gwen de s'émanciper, car, audelà du soutien de Sybil, elle a pu suivre des cours de dactylographie sans l'aide de personne, et chose la plus importante, l'homme qui l'embauche ne le fait pas en raison du soutien qu'elle reçoit de ses employeurs, mais pour les compétences de Gwen et en vertu de son absence de préjugé, puisqu'il vient lui-même des classes populaires et que sa mère elle-même était une femme de chambre. C'est avant tout une solidarité au sein de la classe ouvrière qui permet l'émancipation. Il en va de même avec Sarah Bunting qui transgresse aussi à de nombreuses reprises l'ordre social, elle n'hésite pas à s'opposer frontalement à Robert Crawley et à ses idées et ses discours. Elle se rend ainsi dans les cuisines et à l'étage des domestiques afin de les remercier pour le service et la cuisine. Plus tard, elle se rendra régulièrement dans ce lieu pour contribuer à l'émancipation d'un autre personnage, Daisy, qu'elle n'hésite pas à instruire et à former (Saison 5). Cette dernière finira même par avoir son diplôme (S06E07) grâce au soutien et à l'enseignement de Mr Molesley qui prendra la relève de Sarah Bunting. Ce dernier parvient également à s'émanciper de sa condition de valet. Il était valet de chambre pour Matthew Crawley et perd finalement sa place après la mort de Matthew, avant de redescendre dans la hiérarchie domestique en devenant second valet de pied. Lui qui n'arrivait jamais à franchir les échelons de la hiérarchie domestique et à devenir majordome, finit par faire de sa vocation son métier. La maison lui permet de transgresser sa condition domestique et de rejoindre les enseignants de l'école, d'abord à mi-temps (S06E08), jonglant entre ses travails, avant de rejoindre à plein temps le monde scolaire (S06E09). Cela ne résulte pas de la bonne volonté de l'aristocratie, mais d'une réussite personnelle, il voulait aider Daisy "pour que quelqu'un s'en sorte" (S05E06) et fait un discours similaire à sa classe d'élèves, il se met au service des autres comme agent d'émancipation.

31 L'aristocratie utilise elle aussi les lieux domestiques afin de s'émanciper. Ainsi, lors de son idylle avec Jack Ross, un chanteur noir de jazz, Rose le retrouve et l'embrasse dans la salle des serviteurs (S04E06). Rose s'approprie des lieux éloignés du pouvoir et de l'ordre aristocratiques pour se libérer des contraintes de sa classe sociale. Elle n'hésite également pas à s'habiller en domestique pour rencontrer un homme qui est tombé sous son charme dans un club. Downton regorge de lieux de pouvoirs traditionnels et patriarcaux, mais également de lieux de contre-pouvoir et d'émancipation, permettant, principalement aux femmes de reprendre le contrôle sur elles et leurs vies. Mais il en va de même avec Andrew, jeune valet de pied issu des quartiers populaires de Londres, qui commence à apprendre à lire grâce à Thomas, et quitte également la vie domestique 
pour la vie de ferme qui lui convient davantage, avec l'aide de Mr Mason. La demeure et la série permettent à ces solidarités de se mettre en place, et bien que l'espace appartienne en théorie à l'aristocratie, les classes populaires, principalement, mais également les femmes, se les approprient complètement afin d'arriver à transgresser leur condition, rendant ainsi possible de s'échapper du lieu.

\section{En s'éloignant de la maison}

Un autre moyen d'émancipation dans Downton Abbey est tout simplement l'éloignement de la maison, une opposition et une construction en opposition à l'espace familial, patriarcal, conservateur et aristocratique. Cela passe notamment par la présentation des États-Unis. La famille de Cora, sa mère tout d'abord, Martha Levinson, mais également son frère Harold, fait quelques apparitions dans la série (S03E01 et 02 et S04E09). Harold ne parle ainsi que de son yacht et des soirées qu'il organise, tandis que Martha n'est présentée que pour sa fortune, Violet Crawley faisant tout au long de la série des allusions au manque de raffinement des Américains ${ }^{52}$. Dans la série les personnages américains le rendent bien, et Martha n'hésite pas à affirmer son désaccord. Pour l'actrice qui l'incarne, Shirley MacLaine, ce n'est pas tant la fortune de son personnage, que ses opinions politiques, qui lui dictent son attitude. Pour l'actrice, " son personnage est une démocrate, elle croit énormément en la justice, elle considère ainsi l'Amérique comme juste, et la tradition [britannique] ne peut être juste $»^{53}$. La femme de chambre ne s'y trompe pas, et adopte un comportement émancipé, n'hésitant pas à séduire Alfred, un valet de pied, et allant même jusqu'à l'embrasser. Son explication est donc: " Je suis américaine. Nous sommes en 1920. Il est temps de vivre un peu.» (S03E02). L'espace extérieur, la nation lointaine, apparaît comme une terre d'émancipation, une société permettant de s'élever. Ivy Stuart, l'aide-cuisinière qui arrive au milieu de la troisième saison, et qui parle régulièrement de son désir de ne pas rester cuisinière toute sa vie, à tel point qu'elle est souvent rappelée à l'ordre par Mrs Patmore, fait finalement le choix de s'en aller. Alors qu'une opportunité d'aller aux États-Unis se présente à la fin de la quatrième saison, elle la saisit immédiatement. La série, conservatrice, garde la représentation des États-Unis comme "Terre promise ", mais cela permet une émancipation à certains de ses personnages.

Il n'est pas nécessaire pour autant de partir aussi loin de Downton pour s'émanciper. On peut ainsi citer le cas de Gwen, la femme de chambre devenue secrétaire, qui après son départ de la maison lors de la première saison, y retourne dans la sixième (S06E04). Sauf que, désormais, elle dîne à la table de la famille et demande aux Crawley des financements pour son école de jeunes femmes. Comme Laetitia Kevers le fait remarquer, cette mise en scène permet de signifier que malgré leurs origines sociales la famille Crawley et Gwen poursuivent le même but : construire une école pour les jeunes filles ${ }^{54}$. Néanmoins, elle propose une interprétation uniquement centrée sur l'aristocratie: ce sont les Crawley qui décident de l'école parce que ce sont eux qui financent le projet. Elle nie, par cette analyse, tout l'impact de Gwen, tout son travail pour la mise en place de ce projet et toute son agentivité, elle n'est qu'un personnage des classes populaires supplémentaires. Si l'on regarde à nouveau la scène, c'est elle qui porte le projet, non son mari. Et c'est parce qu'un domestique (Thomas) rappelle qu'elle a travaillé comme domestique dans la maison que la famille parvient à se souvenir d'elle ${ }^{55}$. On peut réellement parler d'émancipation: Gwen est indépendante et mène des projets personnels; tout en gardant une représentation traditionnelle et conservatrice 
de l'aristocratie, qui apparaît nécessaire pour permettre aux classes populaires de financer leurs projets. Le rapport de domination étant explicité et montré l'aristocratie étant même si éloignée de son personnel qu'elle ne parvient pas à reconnaître une ancienne femme de chambre - la scène ne nie alors pas l'agentivité de Gwen, c'est elle qui porte et présente ce projet et les Crawley ne sont qu'un moyen d'y parvenir.

Il en va de même avec Mrs Hughes, l'intendante, lors de son mariage avec Carson le majordome. Elle explique à son fiancé que la réception de leur mariage ne doit pas se passer à Downton (S06E02). Et la série qui présentait des domestiques liés corps et âmes à la famille Crawley, les montres se détacher progressivement, et souhaiter s'éloigner de la maison pour ce faire. Carson tombe ainsi malade au moment où il déménage (S06E09). Il prend son autonomie, tant par sa retraite que par son déménagement. Alors qu'il ne vivait que par et pour son travail, Mrs Hughes parvient à le faire prendre conscience de cela et de l'éloigner de l'influence « néfaste » de la maison sur lui.

Enfin, pour une approche plus genrée, on peut remarquer que plus les femmes s'émancipent plus elles sont hors de la maison, c'est le cas de Sybil qui vit à Leeds puis en Irlande. C'est la plus indépendante des filles et c'est ainsi la première à s'éloigner de la maison, et à être mise en scène en dehors de Downton. Il en va de même pour Edith qui au fur et à mesure de la série développe son caractère, n'hésite pas à s'exprimer dans un journal et à donner ses opinions politiques sur la condition des femmes, elle finit par diriger un journal, et élever sa fille, Marigold, seule. Elle s'éloigne ainsi de la maison, est de plus en plus à Londres, indépendante. C'est progressivement aussi le cas Mary qui à mesure de son émancipation de sa condition de femme (S4 et surtout S5) est en séjour à Londres (hôtel, défilé de mode) très régulièrement, et ne séjourne à Downton que de plus en plus rarement. Enfin, Lady Rosamund (Samantha Bond), la sœur de Robert, sûrement la femme la plus émancipée de la famille et qui conseille régulièrement ses nièces ou s'oppose vivement à sa mère et son frère sur ces questions, vit à Londres, loin de la demeure familiale. Downton, puisqu'elle est une figure d'ordre social et de conservatisme, pousse les personnages qui s'émancipent à s'en éloigner. Les premières étapes de l'émancipation se font en son sein, mais cela ne suffit pas aux personnages féminins qui quittent ensuite cet espace conservateur vers des lieux plus progressistes.

\section{Conclusion}

Downton Abbey présente une demeure protéiforme. Tout à la fois protectrice, conservatrice, émancipatrice, la maison offre différents espaces à ses habitants. Ces derniers peuvent se les approprier pour leur propre bien, lutter contre l'espace conservateur qu'est Downton pour tenter de renverser la hiérarchie sociale, ou comprendre le fonctionnement des lieux afin d'en changer les règles progressivement. Plusieurs personnages, tout comme plusieurs lieux, occupent une fonction de passeurs et de transition entre les différents mondes et les différents espaces. Downton se voit également être le miroir de ces évolutions, suivant le caractère de ses personnages principaux, mais également des évolutions sociales, elle s'ouvre progressivement aux changements. Cette évolution est toutefois trop lente pour certains personnages qui veulent accélérer ces processus, ils sont alors obligés de quitter la demeure pour se construire contre cette dernière, en opposition face au monde qu'elle abrite. Bien que 
la série soit très ouvertement conservatrice, et malgré la vision de l'aristocratie qu'elle propose, cette dernière n'est pas simplement une promotion de ces valeurs comme le suggère Laetitia Kevers ${ }^{56}$, ni une simple héritière des films et séries heritage comme le suggère Katherine Byrne ${ }^{57}$, mais véritablement comme une série post-heritage en ce sens qu'elle fait prendre conscience à ses téléspectateurs que la hiérarchie sociale du début du XXe siècle n'est pas si éloignée de la hiérarchie sociale actuelle. Et enfin, et le plus important de tout: elle est véritablement une série post-heritage ${ }^{58}$ parce qu'elle ne présente pas un simple récit conservateur, brut, et sans adaptation, mais laisse et possède des espaces d'émancipation et de liberté qui sont chéris par ses personnages, auxquels les spectateurs et spectatrices peuvent s'identifier. Downton Abbey peut ainsi être apprécié par toutes et tous alors que l'aristocratie britannique du début du $\mathrm{XX}^{\mathrm{e}}$ siècle aurait toutes les raisons d'éloigner le public.

\section{BIBLIOGRAPHIE}

« Downton Abbey awards », IMDb, https://www.imdb.com/title/tt1606375/awards.

« Downton Abbey US premiere gets record ratings », BBC News, 7 janvier 2014, https:// www.bbc.com/news/entertainment-arts-25634858.

« Lady Almina and The Real Downton Abbey », https://www.highclerecastle.co.uk/lady-alminaand-real-downton-abbey.

ANDERSON, Benedict, L'imaginaire national, Paris, La Découverte, 2006.

BACQUE, Marie-Frédérique, HANUS, Michel, Le deuil, Paris, PUF, 2016.

BAENA, Rosalía, BYKER, Christa, « Dialects of Nostalgia: Downton Abbey and English Identity », National Identities, Vol. 17, No. 3, 2015, p. 259-269.

BARKMAN, Adam, ARP, Robert (dir.), Downton Abbey and Philosophy: Thinking in That Manor, Chicago, Open Court, 2015.

BYRNE, Katherine, Edwardians on Screen: From Downton Abbey to Parade's End, Londres, Palgrave Macmillan, 2015.

BYRNE, Katherine, " Adapting heritage: Class and Conservatism in Downton Abbey ", Rethinking History, Vol. 18, No. 3, 2014, p. 311-327.

CANNADINE, David, The Decline and Fall of the British Aristocracy, New York, Vintage, 1999 [1992].

MISS CELLANIA, « The Rise of the Flapper - Sociological Images », The Society Pages, 25 mars 2013, https://thesocietypages.org/socimages/2013/03/25/guest-post-the-rise-of-the-flapper/.

CHAPMAN, James, « Downton Abbey: Reinventing the British Costume Drama », in British Television Drama, éd. Johnathan Bignell et Stephen Lacey, Londres, Palgrave Macmillan, 2014, p. 131-142. 
CROMPTON, Sarah, « Dan Stevens : Why I Left Downton Abbey », 7 mars 2016, The Telegraph, https://www.telegraph.co.uk/culture/tvandradio/downton-abbey/9765334/Dan-Stevens-Why-Ileft-Downton-Abbey.html.

DE COCK, Laurence, LARRERE, Mathilde, MAZEAU, Guillaume, L'histoire comme émancipation, Paris, Agone, 2019.

DE GROOT, Jerome, Consuming History: Historians and Heritage in Contemporary Popular Culture, Londres, Routledge, 2016 [2009].

DELAP, Lucy, Knowing their Place: Domestic Service in Twentieth-Century Britain, Oxford, Oxford University Press, 2011.

DESANTI, Dominique, La femme au temps des Années Folles, Paris, LGF, 1996.

ENGLISH, Bonnie, A Cultural History of Fashion in the 20th and 21st Centuries. From Catwalk to Sidewalk, Londres, Bloomsbury Academic, (2007) 2013.

FELLOWES, Jessica, FELLOWES, Julian, Downton Abbey: A Celebration - The Official Companion to All Six Seasons, New York, St. Martin's Press, 2015.

FELLOWES, Jessica, FELLOWES, Julian, The World of Downton Abbey, Londres, St. Martin's Press, 2011.

FELLOWES, Jessica, STURGIS, Matthew, FELLOWES, Julian, The Chronicles of Downton Abbey: A New Era, New York, St. Martin's Press, 2012.

FELLOWES, Julian, Downton Abbey Script Book Season 1, New York, William Morrow Paperbacks, 2013.

FERRO, Marc, Cinéma et histoire, Paris, Denoël, 1977.

HALL, Ruth, Passionate Crusader: the Life of Marie Stopes, New York, Harcourt Brace Jovanovich, 1977.

HEWISON, Robert, The Heritage Industry: Britain in a Climate of Decline, Londres, Methuen, 1987.

HIGSON, Andrew, English Heritage, English Cinema: Costume Drama since 1980, Oxford, Oxford University Press, 2003.

IRWIN, William, WHITE, Mark D., Downton Abbey and Philosophy: The Truth Is Neither Here Nor There, Hoboken, John Wiley \& Sons, 2012.

KEVERS, Laetitia, « Re-establishing Class Privilege: The Ideological Uses of Middle and WorkingClass Female Characters in Downton Abbey ", Anglica. An International Journal of English Studies, Vol. 26, No. 1, 2017, p. 221-234.

LEESE, Peter J., Shell Shock: Traumatic Neurosis and the British Soldiers of the First World War, Londres, Palgrave Macmillan, 2002.

LETHBRIDGE, Lucy, Servants: a Downstairs View of Twentieth-Century Britain, Londres, Bloomsbury, 2013.

MONK, Claire, SARGEANT, Amy (dir.), British Historical Cinema: the History, Heritage, and Costume Film, Londres, Routledge, 2002.

PASA, Mario, HINFRAY, Hélène, Instructions et petits secrets du majordome de Downton Abbey pour bien tenir sa maison, Paris, Payot, 2015.

POWELL, Margaret, Servants' Hall: A Real Life Upstairs, Downstairs Romance, St. Martin's Griffin, 2014 [1979]. 
POWELL, Margaret, Below Stairs, Londres, Pan Books, 2011 [1968].

ROSE, June, Marie Stopes and the Sexual Revolution, Londres, Faber \& Faber, 1992.

ROSENSTONE, Robert A., History on Film/Film on History, Londres, Routledge, 2018.

ROSENSTONE, Robert A., « The Historical Film as Real History », Film-Historia, Vol. 5, No. 1, 1995, p. 5-23.

ROSENSTONE, Robert A., « History in Images/History in Words: Reflections on the Possibility of Really Putting History onto Film », The American Historical Review, Vol. 93, No. 5, 1988, p. 1173-1185.

SINGH, Anita, « Downton Abbey sets new viewing record », 4 janvier 2012, The Telegraph, https:// www.telegraph.co.uk/culture/tvandradio/8992602/Downton-Abbey-sets-new-viewingrecord.html.

VERAT, Éric, Génériques : les séries américaines décryptées, Lyon, les Moutons électriques, 2012.

WHITE, Hayden, Metahistory: The Historical Imagination in Nineteenth-Century Europe, Baltimore, Johns Hopkins University Press, 1973.

ZEITZ, Joshua, Flapper: A Madcap Story of Sex, Style, Celebrity, and the Women Who Made America Modern, New York, Crown publishers, 2006.

\section{NOTES}

1. Un peu plus de 11 millions en moyenne en comptant les diffusions sur les plateformes numériques de la chaîne le lendemain de la première diffusion. Anita Singh, «Downton Abbey sets new viewing record », The Telegraph, 4 janvier 2012, https://www.telegraph.co.uk/culture/ tvandradio/8992602/Downton-Abbey-sets-new-viewing-record.html, consulté le 16 avril 2019.

2. Elle a ainsi battu régulièrement les records d'audiences du réseau PBS, notamment pour un premiere ou finale de série avec des audiences autour des 10 millions de téléspectateurs. "Downton Abbey US premiere gets record ratings", BBC News, 7 janvier 2014, https:// www.bbc.com/news/entertainment-arts-25634858, consulté le 16 avril 2019.

3. Avec un score Metacritic de 92 sur 100. Elle a rejoint à ce titre trois séries américaines que sont Mad Men (Matthew Weiner, AMC, 2007 - 2015), Sons of Anarchy (Kurt Suttler, FX, 2008 - 2014) et Modern Family (et Christopher Lloyd (II) et Steven Levitan, ABC, 2009 - en cours).

4. Pour la liste complète des récompenses et nominations de la série dans les différentes cérémonies, voir https://www.imdb.com/title/tt1606375/awards, consulté le 16 avril 2019.

5. Le showrunner est, dans l'univers de la série, la personne responsable du travail quotidien sur une production. Il supervise la réalisation des épisodes, l'écriture du scénario, la composition de la musique, entre autres choses. Il est l'équivalent du réalisateur au cinéma ou du chef d'orchestre en musique.

6. Une grande partie de ces ouvrages est d'ailleurs écrite par Jessica Fellowes, la nièce de Julian Fellowes.

7. Quelques exemples : Jessica Fellowes et Julian Fellowes, The World of Downton Abbey, Londres, St. Martin's Press, 2011; Jessica Fellowes, Matthew Sturgis et Julian Fellowes, The Chronicles of Downton Abbey: A New Era, New York, St. Martin's Press, 2012; Julian Fellowes, Downton Abbey Script Book Season 1, New York, William Morrow Paperbacks, 2013; William Irwin et Mark D. White, Downton Abbey and Philosophy: The Truth Is Neither Here Nor There, Hoboken, John Wiley \& Sons, 2012; Adam Barkman et Robert Arp (dir.), Downton Abbey and Philosophy: Thinking in That Manor, 
Chicago, Open Court, 2015; Mario Pasa et Hélène Hinfray, Instructions et petits secrets du majordome de Downton Abbey pour bien tenir sa maison, Paris, Payot, 2015.

8. Voir par exemple l'article dédié de Katherine Byrne, "Adapting heritage: Class and conservatism in Downton Abbey ", Rethinking History, Vol. 18, No. 3, 2014, p. 311-327.

9. Le terme vient de plusieurs critiques et analyses de films des années 1980 et 1990 . Ces films représentants les débuts $d u$ XXe siècle au Royaume-Uni ont été décrits comme nostalgiques d'une période plus simple, d'une Angleterre des country houses pré-Seconde Guerre mondiale. Voir notamment Robert Hewison, The Heritage Industry: Britain in a Climate of Decline, Londres, Methuen, 1987; Claire Monk et Amy Sargeant (dir.), British Historical Cinema: The History, Heritage, and Costume Film, Londres, New-York, Routledge, 2002.

10. James Chapman, "Downton Abbey: Reinventing the British Costume Drama ", in British Television Drama, éd. Jonathan Bignell et Stephen Lacey, Londres, Palgrave Macmillan, 2014, p. 131-142.

11. Jessica Fellowes et Julian Fellowes, Downton Abbey: A Celebration - The Official Companion to All Six Seasons, New York, St. Martin's Press, 2015, p. 275.

12. Fellowes, Sturgis et Fellowes, p. 9.

13. Hayden white, Metahistory: the historical imagination in nineteenth-century Europe, Baltimore, Johns Hopkins University Press, 1973. Nous parlons de tentative parce qu'il est lui-même revenu une dizaine d'années plus tard sur les conclusions et la méthodologie de son travail, n'en étant pas pleinement satisfait. Sa tentative est toutefois un socle éminent pour cette approche. On peut également mentionner les travaux de Robert Rosenstone, qui depuis les années 1990 mène un travail similaire pour définir les types d'écritures historiques dans les films et documentaires historiques. Robert A. Rosenstone, "The Historical Film as Real History », Film-Historia, Vol. 5, No. 1, 1995, p. 5-23; Robert A. Rosenstone, History on Film/Film on History, Londres, Routledge, 2018.

14. Upstairs, Downstairs (Eileen Atkins et Jean Marsh, ITV, 1971 - 1975).

15. Julian Fellowes s'était déjà essayé au genre, en tant que scénariste, en 2002, avec le film Gosford Park pour lequel il reçoit l'oscar du meilleur scénario, Maggie Smith faisait déjà partie du casting. Robert Altman, Gosford Park, 2002.

16. Rosalía Baena et Christa Byker, " Dialects of nostalgia: Downton Abbey and English identity ", National Identities, Vol. 17, No. 3, 2015, p. 259-269.

17. Nous reprenons ici la définition d'émancipation formulée par Laurence de Cock, Mathilde Larrère et Guillaume Mazeau dans leur ouvrage collectif. L'émancipation n'est pas celle du langage néo-libéral, une sorte de développement personnel, mais davantage une conscientisation des individus, une prise de conscience des rapports de domination. Laurence De Cock, Mathilde Larrère et Guillaume Mazeau, L'histoire comme émancipation, Paris, Agone, 2019.

18. On pourrait même ajouter que c'est un générique décrypteur et d'ambiance. Le générique décrypteur fait fantasmer la série, la symbolise, le générique d'ambiance lui donne le ton d'une série, le son, la musique. Éric Vérat, Génériques: les séries américaines décryptées, Lyon, les Moutons électriques, 2012, p. 41 et 52-53.

19. Comme souvent pour les séries télévisées, et encore plus lorsque celles-ci sont diffusées pendant de nombreuses années, les départs et arrivées d'acteurs et d'actrices sont réguliers. Dans Downton Abbey cela se traduit par la mort abrupte de plusieurs personnages principaux, ou encore même par une disparition soudaine et l'explication par une lettre.

20. Robert ayant dilapidé la majeure partie de la fortune dans des placements financiers non judicieux.

21. Marie-Frédérique Bacqué et Michel Hanus, Le deuil, Paris, PUF, 2016, p. 29-34.

22. Ici nous faisons référence à Anna, la nouvelle femme de Bates.

23. Marie Stopes est une autrice britannique qui a fondé avec son second mari la première clinique de contraception au Royaume-Uni. En 1918 elle publie un manuel de sexe, Married Love, sur comment devrait fonctionner le mariage. Le succès du livre lui permet de publier la même 
année Wise Parenthood: A Book for Married People, un manuel de contraception. Toutefois la contraception prônée par Marie Stopes est empreinte d'eugénisme, et s'adresse en priorité aux classes populaires. Cette dimension est complètement absente de la série qui fait simplement de Marie Stopes une figure féministe œuvrant pour la liberté des femmes. Ruth Hall, Passionate crusader: the life of Marie Stopes, New York, Harcourt Brace Jovanovich, 1977. June Rose, Marie Stopes and the Sexual Revolution, Londres, Faber \& Faber, 1992.

24. Cela correspond toutefois à une réalité historique, relatée par Lucy Lethbridge grâce aux témoignages de certains domestiques dans Servants: a downstairs view of twentieth-century Britain, Londres, Bloomsbury, 2013.

25. Katherine byrne, Edwardians on Screen: From Downton Abbey to Parade's End, Londres, Palgrave Macmillan, 2015, p. 73.

26. On peut citer les séries comme Upstairs, Downstairs (Eileen Atkins et Jean Marsh, ITV, 1971-1975) ou The Forsyte Saga (David Gilles et James Cellan Jones BBC Two, 1967) pour le XX siècle, toutes deux ont d'ailleurs eu une nouvelle adaptation, The Forsyte Saga (Christopher Menaul, David Moore et Andy Wilson, ITV, 2002-2003) et Upstairs, Downstairs (Heidi Thomas, BBC, 2010-2012).

27. Cela change ensuite pour les saisons suivantes et les chambres sont également construites à l'identique dans les studios Ealing. Fellowes, Sturgis et Fellowes, p. 307-308.

28. Fellowes et Fellowes, p. 29.

29. Cette opposition entre above et below reprend exactement les mêmes procédés qu'entre upstairs/downstairs, nous avons fait le choix ici de rester au plus proche du texte d'origine.

30. Fellowes et Fellowes, p 281.

31. Notamment du pilote de la série.

32. Fellowes, Sturgis et Fellowes, p. 304.

33. Byrne, p. 70.

34. Byrne, p. 77.

35. Comprendre par là qu'elle est mère célibataire et doit se prostituer pour subvenir aux besoins de son fils.

36. La première femme de Bates est en effet présentée comme calculatrice et cherchant avant tout à exploiter son mari qui tente de lui échapper. Par un concours de circonstances, elle finit par apprendre l'aventure de Mary avec Kemal Pamuk et utilise alors ce levier pour faire céder à son chantage Bates, qui souhaitait divorcer.

37. Ici une fausse couche provoquée par O’Brien, pensant être renvoyée et souhaitant se venger de Cora.

38. On peut notamment se référer à ces deux ouvrages pour une analyse de la situation domestique au début $\mathrm{du} \mathrm{XX}^{\mathrm{e}}$ siècle : Lucy Delap, Knowing their place: domestic service in twentiethcentury Britain, Oxford, Oxford University Press, 2011 ; Lethbridge, op. cit.

39. Benedict Anderson, L'imaginaire national, Paris, La Découverte, 2006.

40. Sarah Crompton, «Dan Stevens : Why I Left Downton Abbey », The Telegraph, 7 mars 2016, https://www.telegraph.co.uk/culture/tvandradio/downton-abbey/9765334/Dan-Stevens-Why-Ileft-Downton-Abbey.html, consulté le 12 avril 2019.

41. David Cannadine, The Decline and fall of the British aristocracy, $2^{\text {nde }}$ éd., New York, Vintage, 1999, p. 75-76.

42. "Lady Almina and The Real Downton Abbey ", https://www.highclerecastle.co.uk/ladyalmina-and-real-downton-abbey, consulté le 14 avril 2019.

43. Peter J. Leese, Shell shock: traumatic neurosis and the British soldiers of the First World War, Londres, Palgrave Macmillan, 2002, p. 21.

44. Laetitia Kevers, « Re-establishing Class Privilege: The Ideological Uses of Middle and WorkingClass Female Characters in Downton Abbey », Anglica. An International Journal of English Studies, Vol. 26, No. 1, 2017, p. 231 [p. 221-234]. 
45. Musique récurrente dans la série, elle habille le fond sonore de diverses manigances, notamment de Thomas et de Miss O'Brien.

46. Laurence De Cock, Mathilde Larrère et Guillaume Mazeau, L'histoire comme émancipation, Paris, Agone, 2019, p. 81.

47. Rejoignant ainsi les travaux fondateurs de Robert Rosenstone et de Marc Ferro pour les films historiques (Rosenstone, op. cit.; Marc FERRO, Cinéma et histoire, Nouvelle ed. (1977)., Paris, Gallimard, 1993).

48. Kevers, p. 222.

49. Très mal vu pendant une grande partie du $\mathrm{XX}^{\mathrm{e}}$ siècle, le port du pantalon par les femmes a été un combat, progressivement les ouvrières ont réussi à l'imposer pour leur travail à l'usine dans les années 20. Le pantalon que porte Sybil est une référence à l'introduction du sarouel dans la mode parisienne par Paul Poiret en 1910. Bonnie English, A Cultural History of Fashion in the 20th and 21st Centuries. From Catwalk to Sidewalk, Londres, Bloomsbury, 2013, p. 23.

50. Cette mode à la garçonne, ou Flapper est caractéristique des années 1920, aussi appelées Roaring Twenties ou Années folles. Ce style de coupe, Dutch bob ou Pageboy bob est devenu symbolique de cette décennie, à ce titre il est présent dans la majorité des films et séries sur la période, à travers au moins une des personnages. Dominique Desanti, La femme au temps des Années folles, Paris, LGF, 1996. Miss Cellania, "The Rise of the Flapper - Sociological Images ", The Society Pages, 25 mars 2013, https://thesocietypages.org/socimages/2013/03/25/guest-post-therise-of-the-flapper/, consulté le 10 juillet 2019. Joshua Zeitz, Flapper: a madcap story of sex, style, celebrity, and the women who made America modern, New York, Crown Publishers, 2006.

51. Kevers, p. 225.

52. Lors d'une dispute elle dit ainsi « c'est ce qu'on appelle une discussion à New York » (S01E01), ou encore qu'elle aime être en présence de Martha puisque cela lui permet de se souvenir des vertus britanniques (S03E01).

53. Fellowes, Sturgis et Fellowes, p. 105.

54. Kevers, p. 224.

55. En effet, comme le raconte Margaret Powell dans ses romans et Lucy Delap l'explique dans les familles aristocratiques la hiérarchie sociale était établie par des noms et des uniformes. De nombreux domestiques voient leur nom modifié en entrant en service, certaines familles avaient des noms prédéfinis pour les valets de pied, selon leur position. De plus, les femmes de chambre se devaient, à l'inverse de ce qui est représenté dans Downton Abbey, d'être invisibles pour la famille. Dans Downton Abbey cela apparaît davantage comme un oubli de bonne foi, probablement lié au temps écoulé depuis le départ de Gwen. Voir Delap, op. cit. Margaret Powell, Servants' Hall: A Real Life Upstairs, Downstairs Romance, St. Martin's Griffin, 2014 [1979]. Margaret Powell, Below Stairs, Unabridged edition, Londres, Pan Books, 2011 [1968].

56. Kevers, p. 222.

57. Byrne, p. 87.

58. En reprenant la définition du post-heritage par Jerome De Groot (Consuming history: historians and heritage in contemporary popular culture, Londres, Routledge, 2009, p. 184). 


\section{RÉSUMÉS}

Pendant 6 saisons, la série Downton Abbey (ITV1, 2010-2015) a raconté l'histoire, au début du XXe siècle, de la famille Crawley, issue de l'aristocratie britannique, et de ses domestiques. Le titre de la série, ainsi que l'image qui accompagne le générique, font porter l'attention, non pas tant sur les personnages eux-mêmes que sur le lieu où ils demeurent et où l'essentiel de l'action se déroule. Tout cela comme si la maison, la demeure familiale, était l'héroïne de la série, dont les personnages ne seraient alors que des résidents temporaires.

Dans la maison, d'un étage à l'autre, certains personnages se reflètent et se répondent, quel que soit leur milieu d'origine. C'est la proximité de ces caractères et de ces personnages, au cœur de la demeure familiale, qui forme le sujet de la série. La maison fonctionne ainsi comme une métaphore, notamment de la nation, et son microcosme interne permet de développer une vision du Royaume-Uni. Mais la série reproduit également des rapports historiques ou semblables historiquement, à la demeure, à l'ordre ou encore à la hiérarchie sociale.

Les rapports des personnages avec la demeure forment le sujet de cette analyse. Comprendre les rapports qu'ils entretiennent avec les différents espaces qui composent Downton permet de faire ressortir des sujets et des méthodes d'écritures de l'Histoire. Malgré une vision conservatrice, assumée et cultivée, la demeure révèle non seulement ces proximités de caractères, mais est également un lieu de rencontre, voire de passage d'une classe à une autre. Certains espaces ainsi que certains personnages sont des moyens d'émancipations et sont sollicités à de nombreuses reprises. D'autres personnages se construisent ou évoluent en opposition au lieu familial. Analyser tous ces fonctionnements permet de comprendre les forces qui régissent le monde de la série.

For 6 series, Downton Abbey (ITV1, 2010-2015) narrated the story of the aristocratic Crawley family and of their domestic servants, in the early $20^{\text {th }}$ century. The series' title and opening credits focus on the country estate where the narrative unfolds, making the 'big house' the main character of the series.

In the mansion, characters interact within and between their own social/geographic areas in an "upstairs, downstairs" logic. The drama that develops at the scale of the house reflects the social and historical tensions of the United Kingdom before and after the First World War. This article examines the characters' complex relationships with the spaces and places that compose the Big House, in order to reassess Julian Fellowes' conservative view of class and emancipation in the fictional universe of the series.

\section{INDEX}

Mots-clés : Downton Abbey, upstairs downstairs, émancipation, espace, écriture de l'Histoire, femmes, conservatisme, demeure, heritage, country houses, Fellowes Julian

Keywords : Downton Abbey, upstairs downstairs, emancipation, space, writing of History, women, conservatism, dwelling, heritage, country houses, Fellowes Julian 


\section{AUTEUR}

\section{VICTOR FAINGNAERT}

Victor Faingnaert is a PhD student in contemporary History at the University of Caen Normandie. His research focuses on the writing of History and on how History is passed on through fiction, especially in TV series. After a master's thesis on the TV show Rome ("An historical fiction Between references and historical researches: the TV show Rome"), he's extending his research in a thesis focusing on the aftermath of the First World War in the United Kingdom in TV shows. Thus, his work is about historical imaginaries which stand out in those times of memorial moments of the centenary.

Victor Faingnaert est doctorant en Histoire contemporaine à l'Université de Caen Normandie. Il s'intéresse à l'écriture de l'Histoire et à sa mise en fiction, principalement dans les séries télévisées. Il travaille ainsi sur la transmission de l'Histoire sous forme fictionnelle. Après un mémoire sur la série Rome (« Une fiction historique - Entre références et recherches historiques : la série Rome »), il prolonge aujourd'hui ses recherches à travers une thèse centrée sur l'aprèsPremière Guerre mondiale au Royaume-Uni dans les séries télévisées. Son travail porte ainsi sur les imaginaires sociaux qui ressortent en cette période de moment mémoriel et du centenaire. 